\title{
Global behavior of solutions to chevron pattern equations
}

Cite as: J. Math. Phys. 61, 061511 (2020); https://doi.org/10.1063/5.0012525

Submitted: 01 May 2020 . Accepted: 26 May 2020 . Published Online: 17 June 2020

H. Kalantarova (iD, V. Kalantarov, and O. Vantzos

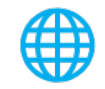

View Online

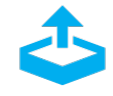

Export Citation

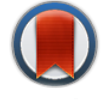

CrossMark

\section{ARTICLES YOU MAY BE INTERESTED IN}

Asymptotic profiles of solutions to the generalized double dispersion equation with structural damping

Journal of Mathematical Physics 61, 061507 (2020); https://doi.org/10.1063/1.5138673

Ground states of two-component Bose-Einstein condensates passing an obstacle Journal of Mathematical Physics 61, 061501 (2020); https://doi.org/10.1063/5.0004998

Decay of regular solutions for the critical 2D Zakharov-Kuznetsov equation posed on rectangles

Journal of Mathematical Physics 61, 061509 (2020); https://doi.org/10.1063/1.5100284

Journal of

Mathematical Physics

Special Issue: XIXth International Congress on Mathematical Physics 


\title{
Global behavior of solutions to chevron pattern equations
}

\author{
Cite as: J. Math. Phys. 61, 061511 (2020); doi: 10.1063/5.0012525 \\ Submitted: 1 May 2020 - Accepted: 26 May 2020 • \\ Published Online: 17 June 2020
}

\section{H. Kalantarova, ' (D) V. Kalantarov, ${ }^{2, a), b)}$ and O. Vantzos}

\author{
AFFILIATIONS \\ ${ }^{1}$ Department of Material Science and Engineering, Technion-Israel Institute of Technology, 32000 Haifa, Israel \\ ${ }^{2}$ Department of Mathematics, Koç University, Istanbul, Turkey \\ ${ }^{3}$ Lightricks Ltd., Jerusalem, Israel
}

a) Author to whom correspondence should be addressed: vkalantarov@ku.edu.tr
b) Also at: Azerbaijan State Oil and Industry University, Baku, Azerbaijan.

\begin{abstract}
Considering a system of equations modeling the chevron pattern dynamics, we show that the corresponding initial boundary value problem has a unique weak solution that continuously depends on initial data, and the semigroup generated by this problem in the phase space $X^{0}:=L^{2}(\Omega) \times L^{2}(\Omega)$ has a global attractor. We also provide some insight into the behavior of the system, by reducing it under special assumptions to systems of ordinary differential equations, which can, in turn, be studied as dynamical systems.
\end{abstract}

Published under license by AIP Publishing. https://doi.org/10.1063/5.0012525

\section{INTRODUCTION}

The chevron patterns, also known as the herringbone patterns in the context of the electroconvection of nematic liquid crystals, i.e., in the electromagnetically driven motion of anisotropic liquids composed of rod-like particles, which can be oriented freely in space, were first studied by Heilmeier and Helfrich ${ }^{1}$ and then in detail by the Orsay group. ${ }^{2}$ The typical experimental setup involves, in general, the containment of the nematic liquid between two parallel transparent plates and the application of an AC voltage of varying frequency across the plates, often in conjunction with a magnetic field parallel to the plates (see Fig. 1). Depending on the characteristics (voltage and frequency) of the external driving forces, the behavior of the fluid exhibits a wealth of nonlinear dynamical phenomena (Refs. 3-7). A common class of such phenomena feature the self-organization of the nematic liquid into convection cells, where the flow is regular and largely local to each cell. The formation of these cells is driven on a microscopic level by the interaction of the external forces with the constituent particles of the liquid, and therefore, the orientation of the particles is of great importance to the dynamics. The theoretical study of this problem focuses then mainly on macroscopic models that attempt to capture directly the distribution and flow pattern (such as direction and orientation of rotation) of the convection cells, coupled with a measure of the local average orientation of the fluid particles (for instance, in the form of a so-called director vector field).

We are interested, in particular, in the case where the fluid flow takes the form of rolls, i.e., zones where the fluid rotates parallel to the plates; the rolls themselves are arranged in periodical configurations, alternating between clockwise and counterclockwise rotations (see Fig. 2). The periodicity of the rolls does not hold on larger scales, which leads to interesting formations such as the titular chevron pattern, where two sequences of alternating rolls meet at an angle (see Fig. 3). The following system of equations was proposed by Rossberg et al. ${ }^{8,9}$ to model the evolution of such patterns:

$$
\begin{aligned}
& \tau \partial_{t} A=A+\Delta A-\phi^{2} A-|A|^{2} A-2 i c_{1} \phi \partial_{y} A+i \beta A \partial_{y} \phi, \\
& \partial_{t} \phi=D_{1} \partial_{x}^{2} \phi+D_{2} \partial_{y}^{2} \phi-h \phi+\phi|A|^{2}-c_{2} \operatorname{Im}\left[A^{*} \partial_{y} A\right]
\end{aligned}
$$




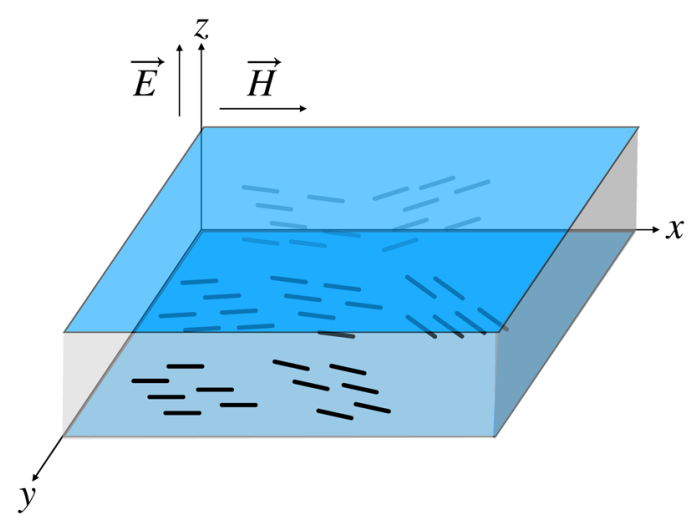

FIG. 1. Cross section of a nematic liquid crystal contained between two parallel transparent plates. The liquid is composed of rod-like particles, which are free to flow and orient themselves in the 3D space, under the influence of external electric $(\vec{E})$ and magnetic $(\vec{H})$ fields.

where $\tau, D_{1}, D_{2}, c_{1}, c_{2}$, and $h$ are non-negative constants and $\beta \in \mathbb{R}$. The complex valued function $A$ (where $A^{*}$ denotes its complex conjugate) succinctly represents the phase (clockwise/counterclockwise), direction, and amplitude (wave vector) of the periodical patterns, whereas the orientation of the liquid crystals is represented via the real valued function $\phi$, the angle of the director vector (projected in the $x-y$ plane) with the $\mathrm{x}$-axis. The parameter $\tau$ is a function of the various physical timescales of the problem, and $D_{1}$ and $D_{2}$ are the coefficients of the anisotropic diffusion of the director field for the liquid crystal particles. The rest of the parameters reflect various interactions:

- the dampening parameter $h$ measures the tendency of the director field to align with the magnetic field $\vec{H}$, corresponding to $\phi=0$;

- the parameter $\beta$ measures the interaction between the gradient of the director field and the phase of the rolls; and

- the parameters $c_{1}$ and $c_{2}$ control the torque that the director field and the wave vector of the rolls exert on each other; when $c_{1}=c_{2}=1$, the interaction is isotropic, but many experimentally interesting phenomena occur in the anisotropic regime.

In the literature, one can mostly find experimental (Refs. 10 and 11) and numerical (Refs. 12 and 13) studies on chevron patterns and various works on the physical derivation of the model (Refs. 8, 9, and 14). It is also interesting to apply mathematical analysis tools to study this type of problem, which models a physical phenomenon that features rich nonlinear behavior. In our work, we show the existence, uniqueness, and the continuous dependence on the initial data for the weak solutions of the model. Moreover, we show the existence of a global attractor of a semigroup generated by this problem. These results are valid under the assumption that the parameter $c_{1}$ is in the range $[0,1)$.

The rest of this paper is organized as follows: In Sec. II, we prove the well-posedness of the initial boundary value problem for the system (1) and (2) and dissipativity of the semigroup generated by this problem, provided that the coefficient of the nonlinear term $2 i \phi \partial_{y} A$ in (1) is restricted to the range $0 \leq c_{1}<1$. In Sec. III, we show that the semigroup generated by the problem is a compact semigroup and has a global attractor under the same assumption $c_{1} \in[0,1)$. In Sec. IV, we present an argument, by reducing the model to a dynamical system,

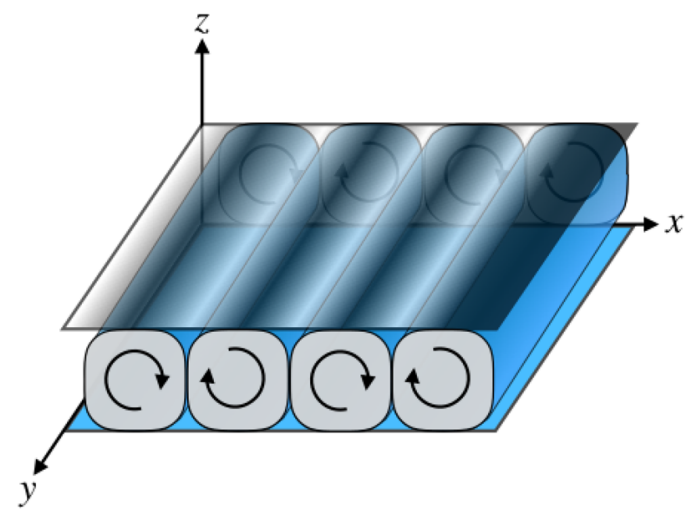

FIG. 2. A typical flow pattern where the liquid self-organizes into rotating zones, called rolls, with the axes of rotation parallel to the plates and rotation orientation alternating between clockwise and counterclockwise. 


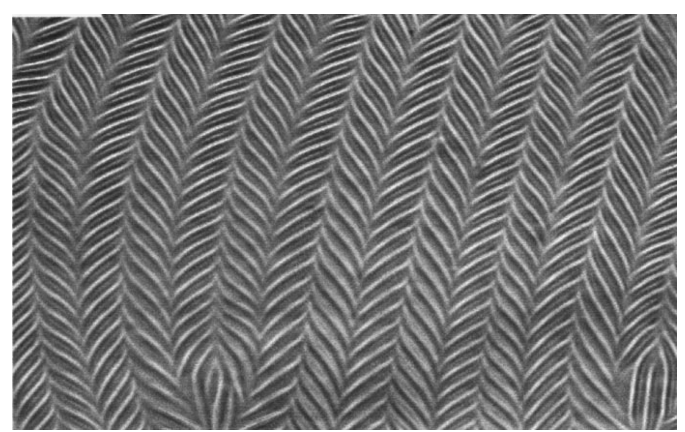

FIG. 3. Experimental observation of chevron patterns, where periodic groups of rolls (observable as alternating light-dark zones) meet at an angle. Reprinted with permission from Fig. 2(a) Huh et al., Phys. Rev. E 61, 2769 (2000). Copyright 2000 by the American Physical Society.

which gives insight and supports the assumed condition on the parameter $c_{1}$ by showing that $c_{1}=1$ is a critical value for the dynamics of the system.

Notation. Throughout this paper, $\mathbb{R}$ denotes the set of real numbers and $\Omega \subset \mathbb{R}^{2}$ denotes a bounded domain with a sufficiently smooth boundary denoted by $\partial \Omega$.

$(\cdot, \cdot)$ and $\|\cdot\|$ denote the inner product and the norm induced by it in $L^{2}(\Omega)$, respectively. That is, for $f, g \in L^{2}(\Omega)$,

$$
(f, g):=\int_{\Omega} f(x, y) g^{*}(x, y) d x d y, \quad\|f\|:=\left(\int_{\Omega}|f(x, y)|^{2} d x d y\right)^{1 / 2}
$$

\section{EXISTENCE, UNIQUENESS, AND DISSIPATIVITY}

In this section, we study the system (1) and (2) in $\Omega \subset \mathbb{R}^{2}$ under the following initial and boundary conditions:

$$
\left.A\right|_{t=0}=A_{0},\left.\quad \phi\right|_{t=0}=\phi_{0},\left.\quad A\right|_{\partial \Omega}=0,\left.\quad \phi\right|_{\partial \Omega}=0,
$$

where $A_{0}, \phi_{0} \in L^{2}(\Omega)$ are given functions.

To prove the existence and uniqueness of a weak solution of the problem, we need two a priori estimates. To find these estimates, we multiply equation (1) by $A^{*}$ and we multiply equation (2) by $\phi$ and integrate them with respect to $x$ over $\Omega$. Then, we take the real parts of the resulting identities and obtain the following inequalities:

$$
\begin{gathered}
\frac{\tau}{2} \frac{d}{d t}\|A\|^{2}-\|A\|^{2}+\|\nabla A\|^{2}+\left(\phi^{2},|A|^{2}\right)+\|A\|_{L^{4}}^{4} \leq 2 c_{1}\left|\left(\phi \partial_{y} A, A\right)\right|, \\
\frac{1}{2} \frac{d}{d t}\|\phi\|^{2}+D_{1}\left\|\partial_{x} \phi\right\|^{2}+D_{2}\left\|\partial_{y} \phi\right\|^{2}+\left(h, \phi^{2}\right) \leq c_{2}\left|\left(A^{*} \partial_{y} A, \phi\right)\right|+\left(|\phi|^{2},|A|^{2}\right),
\end{gathered}
$$

respectively, since $c_{1}, c_{2}$ are non-negative constants. We get

$$
\begin{aligned}
& 2 c_{1}\left|\left(\phi \partial_{y} A, A\right)\right| \leq c_{1}\left\|\partial_{y} A\right\|^{2}+c_{1}\left(|A|^{2}, \phi^{2}\right), \\
& c_{2}\left|\left(A^{*} \partial_{y} A, \phi\right)\right| \leq \frac{1}{2} c_{2}\left\|\partial_{y} A\right\|^{2}+\frac{1}{2} c_{2}\left(|A|^{2} \phi^{2}\right),
\end{aligned}
$$

upon application of Hölder's inequality and then Cauchy's inequality. Employing the inequality (6) in (4) and the inequality (7) in (5), we obtain

$$
\frac{\tau}{2} \frac{d}{d t}\|A\|^{2}-\|A\|^{2}+\left(1-c_{1}\right)\left\|\partial_{y} A\right\|^{2}+\left\|\partial_{x} A\right\|^{2}+\left(1-c_{1}\right)\left(\phi^{2},|A|^{2}\right)+\|A\|_{L^{4}(\Omega)}^{4} \leq 0
$$

and 


$$
\frac{1}{2} \frac{d}{d t}\|\phi\|^{2}+D_{1}\left\|\partial_{x} \phi\right\|^{2}+D_{2}\left\|\partial_{y} \phi\right\|^{2}+\left(h, \phi^{2}\right) \leq \frac{1}{2} c_{2}\left\|\partial_{y} A\right\|^{2}+\left(1+\frac{1}{2} c_{2}\right)\left(|\phi|^{2},|A|^{2}\right),
$$

respectively. Next, we multiply the inequality (9) by a parameter $\delta>0$, whose value is to be determined later, and add it to (8),

$$
\begin{aligned}
\frac{1}{2} \frac{d}{d t}\left[\tau\|A\|^{2}+\delta\|\phi\|^{2}\right] & +\delta D_{1}\left\|\partial_{x} \phi\right\|^{2}+\delta D_{2}\left\|\partial_{y} \phi\right\|^{2}+\delta h\|\phi\|^{2}-\|A\|^{2}+\left\|\partial_{x} A\right\|^{2} \\
+ & \left(1-c_{1}-\frac{1}{2} \delta c_{2}\right)\left\|\partial_{y} A\right\|^{2}+\left(1-c_{1}-\delta\left(1+\frac{1}{2} c_{2}\right)\right)\left(\phi^{2},|A|^{2}\right)+\|A\|_{L^{4}(\Omega)}^{4} \leq 0
\end{aligned}
$$

Choosing

$$
\delta=\delta_{0}:=\frac{2\left(1-c_{1}\right)}{2+c_{2}}, \quad \text { where } \quad c_{1}<1,
$$

we optimize the range of values of $c_{1}$ and the number of terms with non-negative coefficients in the above inequality, which yields

$$
\begin{aligned}
\frac{1}{2} \frac{d}{d t}\left[\tau\|A\|^{2}+\delta_{0}\|\phi\|^{2}\right]+\delta_{0} D_{1}\left\|\partial_{x} \phi\right\|^{2}+ & \delta_{0} D_{2}\left\|\partial_{y} \phi\right\|^{2} \\
& +\delta_{0} h\|\phi\|^{2}-\|A\|^{2}+\left\|\partial_{x} A\right\|^{2}+\delta_{0}\left\|\partial_{y} A\right\|^{2}+\|A\|_{L^{4}}^{4} \leq 0 .
\end{aligned}
$$

Using the inequality $2\|A\|^{2} \leq|\Omega|+\|A\|_{L^{4}(\Omega)}^{4}$, where $|\Omega|$ denotes the measure of the domain $\Omega$, on the last term in (10), we get

$$
\frac{1}{2} \frac{d}{d t}\left[\tau\|A\|^{2}+\delta_{0}\|\phi\|^{2}\right]+\|A\|^{2}+\delta_{0} h\|\phi\|^{2}+\delta_{0}\|\nabla A\|^{2}+\delta_{0} D_{0}\|\nabla \phi\|^{2} \leq|\Omega|,
$$

where $D_{0}=\min \left\{D_{1}, D_{2}\right\}$. It follows from (11) that

$$
\frac{d}{d t}\left[\tau\|A\|^{2}+\delta_{0}\|\phi\|^{2}\right]+k_{0}\left[\tau\|A\|^{2}+\delta_{0}\|\phi\|^{2}\right] \leq|\Omega|,
$$

where $k_{0}=\min \left\{\tau^{-1}, h\right\}$. Integrating the last inequality, we get

$$
\tau\|A(t)\|^{2}+\delta_{0}\|\phi(t)\|^{2} \leq\left[\tau\left\|A_{0}\right\|^{2}+\delta_{0}\left\|\phi_{0}\right\|^{2}\right] e^{-k_{0} t}+\frac{1}{k_{0}}|\Omega| .
$$

By deriving a priori estimates (11) and (12), we have proved

$$
\|\phi(t)\| \leq M_{0}, \quad\|A(t)\| \leq M_{0}, \quad \forall t>0,
$$

and

$$
\int_{0}^{T}\|\nabla \phi(t)\|^{2} d t \leq M_{T}, \quad \int_{0}^{T}\|\nabla A(t)\|^{2} d t \leq M_{T} .
$$

Here and in what follows, $M_{0}$ denotes a generic constant, depending only on $\left\|A_{0}\right\|,\left\|\phi_{0}\right\|$, and $|\Omega| ; M_{T}$ is a generic constant, which depends on $T,\left\|A_{0}\right\|,\left\|\phi_{0}\right\|$, and $|\Omega|$. The estimates (13) and (14) allow us by using the standard Galerkin method to prove the existence of a weak solution of the problem. Furthermore, employing the estimates (13) and (14), we can now prove that the solution of the problem is unique. Suppose that $[A, \phi]$ and $[\tilde{A}, \tilde{\phi}]$ are solutions of the system (1) and (2) corresponding to initial data $\left[A_{0}, \phi_{0}\right]$ and $\left[\tilde{A}_{0}, \tilde{\phi}_{0}\right]$, respectively, then $[a, \Phi]:=[A-\tilde{A}, \phi-\tilde{\phi}]$ is a solution of the system

$$
\begin{gathered}
\tau \partial_{t} a=a+\Delta a-\phi^{2} a-\left(\phi^{2}-\tilde{\phi}^{2}\right) \tilde{A}-|A|^{2} A+|\tilde{A}|^{2} \tilde{A}-2 i c_{1}\left[\Phi \partial_{y} A+\tilde{\phi} \partial_{y} a\right]+i \beta\left[a \partial_{y} \phi+\tilde{A} \partial_{y} \Phi\right], \\
\partial_{t} \Phi=D_{1} \partial_{x}^{2} \Phi+D_{2} \partial_{y}^{2} \Phi-h \Phi+\Phi|A|^{2}+\tilde{\phi}\left(|A|^{2}-|\tilde{A}|^{2}\right)-c_{2} \operatorname{Im}\left[a^{*} \partial_{y} A+\tilde{A}^{*} \partial_{y} a\right] .
\end{gathered}
$$

Next, we multiply equation (15) by $a^{*}$ and equation (16) by $\Phi$ in $L^{2}(\Omega)$ and get

$$
\begin{aligned}
\frac{1}{2} \frac{d}{d t}\left[\tau\|a\|^{2}\right. & \left.+\|\Phi\|^{2}\right]-\|a\|^{2}+\|\nabla a\|^{2}+\left(\phi^{2},|a|^{2}\right)+D_{0}\|\nabla \Phi\|^{2}+h\|\Phi\|^{2} \\
\leq & |((\phi+\tilde{\phi}) \Phi \tilde{A}, a)|+\left(|A \| \tilde{A}|,|a|^{2}\right)+\left(|\tilde{A}|^{2},|a|^{2}\right)+2 c_{1}\left(\left|\Phi \| \partial_{y} A\right|,\left|a^{*}\right|\right) \\
& +2 c_{1}\left(\left|\tilde{\phi} \| \partial_{y} a\right|,\left|a^{*}\right|\right)+\beta\left(\left|\tilde{A} \| \partial_{y} \Phi\right|,\left|a^{*}\right|\right)+\left(\Phi^{2},|A|^{2}\right)+|(\tilde{\phi} \Phi,(|A|+|\tilde{A}|)|a|)| \\
& +c_{2}\left|\left(a^{*} \partial_{y} A, \Phi\right)\right|+c_{2}\left|\left(\tilde{A}^{*} \partial_{y} a, \Phi\right)\right|
\end{aligned}
$$


where $D_{0}=\min \left\{D_{1}, D_{2}\right\}$, as in (11). We will estimate each term on the right-hand side of the inequality (17) by employing (13), Hölder's inequality, Cauchy's inequality, and the Ladyzhenskaya inequality,

$$
\|u\|_{L^{4}(\Omega)} \leq 2^{1 / 4}\|u\|^{1 / 2}\|\nabla u\|^{1 / 2},
$$

which is valid for each function $u \in H_{0}^{1}(\Omega)$ with $\Omega \subset \mathbb{R}^{2}$. We start with

$$
\begin{aligned}
|(\phi \Phi \tilde{A}, a)| & \leq\|\phi\|_{L^{4}(\Omega)}\|\Phi\|_{L^{4}(\Omega)}\|\tilde{A}\|_{L^{4}(\Omega)}\|a\|_{L^{4}(\Omega)} \\
& \leq \frac{1}{2}\|\phi\|_{L^{4}(\Omega)}^{2}\|\Phi\|_{L^{4}(\Omega)}^{2}+\frac{1}{2}\|\tilde{A}\|_{L^{4}(\Omega)}^{2}\|a\|_{L^{4}(\Omega)}^{2} \\
& \leq\|\phi\|\|\nabla \phi\|\|\Phi\|\|\nabla \Phi\|+\|\tilde{A}\|\|\nabla \tilde{A}\|\|a\|\|\nabla a\| \\
& \leq \varepsilon_{1}\|\nabla \Phi\|^{2}+\varepsilon_{1}\|\nabla a\|^{2}+\frac{M_{0}^{2}}{4 \varepsilon_{1}}\left[\|\nabla \phi\|^{2}\|\Phi\|^{2}+\|\nabla \tilde{A}\|^{2}\|a\|^{2}\right]
\end{aligned}
$$

which gives us the estimate of the first term on the right-hand side of (17),

$$
\begin{aligned}
|((\phi+\tilde{\phi}) \Phi \tilde{A}, a)| \leq 2 \varepsilon_{1}\left[\|\nabla \Phi\|^{2}+\|\nabla a\|^{2}\right] & \\
& +\frac{M_{0}^{2}}{4 \varepsilon_{1}}\left[\|\nabla \phi\|^{2}\|\Phi\|^{2}+\|\nabla \tilde{\phi}\|^{2}\|\Phi\|^{2}+2\|\nabla \tilde{A}\|^{2}\|a\|^{2}\right] .
\end{aligned}
$$

Then, we estimate the second term through the fourth term as follows:

$$
\begin{aligned}
\left(|A \| \tilde{A}|,|a|^{2}\right) & \leq\|A\|_{L^{4}}\|\tilde{A}\|_{L^{4}}\|a\|_{L^{4}}^{2} \\
& \leq 2\|A\|^{1 / 2}\|\nabla A\|^{1 / 2}\|\tilde{A}\|^{1 / 2}\|\nabla \tilde{A}\|^{1 / 2}\|a\|\|\nabla a\| \\
& \leq\|A\|\|\nabla A\|\|a\|\|\nabla a\|+\|\tilde{A}\|\|\nabla \tilde{A}\|\|a\|\|\nabla a\| \\
& \leq 2 \varepsilon_{2}\|\nabla a\|^{2}+\frac{M_{0}^{2}}{4 \varepsilon_{2}}\left[\|\nabla A\|^{2}\|a\|^{2}+\|\nabla \tilde{A}\|^{2}\|a\|^{2}\right], \\
\left(|\tilde{A}|^{2},|a|^{2}\right) & \leq\|\tilde{A}\|_{L^{4}}^{2}\|a\|_{L^{4}}^{2} \\
& \leq 2\|\tilde{A}\|\|\nabla \tilde{A}\|\|a\|\|\nabla a\| \\
& \leq \varepsilon_{3}\|\nabla a\|^{2}+\frac{M_{0}^{2}}{4 \varepsilon_{3}}\|\nabla \tilde{A}\|^{2}\|a\|^{2}, \\
2 c_{1}\left(|\Phi| \| \partial_{y} A|,| a \mid\right) \leq 2 c_{1}\|\Phi\|_{L^{4}}\|\nabla A\|\|a\|_{L^{4}} & \leq 2 \sqrt{2} c_{1}\|\Phi\|^{1 / 2}\|\nabla \Phi\|^{1 / 2}\|\nabla A\|\|a\|^{1 / 2}\|\nabla a\|^{1 / 2} \\
& \leq \sqrt{2} c_{1}\|\Phi\|\|\nabla \Phi\|\|\nabla A\|+\sqrt{2} c_{1}\|\nabla A\|\|a\|\|\nabla a\| \\
& \leq \varepsilon_{4}\left[\|\nabla \Phi\|^{2}+\|\nabla a\|^{2}\right]+\frac{c_{1}^{2}}{2 \varepsilon_{4}}\|\nabla A\|^{2}\left[\|a\|^{2}+\|\Phi\|^{2}\right] .
\end{aligned}
$$

We can similarly estimate the other terms on the right-hand side of (17), and properly choosing the positive parameters $\varepsilon_{i}$ in these estimates, we get the following inequality:

$$
\frac{d}{d t}\left[\tau\|a\|^{2}+\|\Phi\|^{2}\right] \leq K_{0} \mathscr{E}(t)\left[\tau\|a\|^{2}+\|\Phi\|^{2}\right],
$$

where

$$
\mathscr{E}(t)=\|\nabla A(t)\|^{2}+\|\nabla \phi(t)\|^{2}+\|\nabla \tilde{A}(t)\|^{2}+\|\nabla \tilde{\phi}(t)\|^{2} .
$$

Integrating this inequality and remembering (14), we obtain 


$$
\tau\|A(t)-\tilde{A}(t)\|^{2}+\|\phi(t)-\tilde{\phi}(t)\|^{2} \leq e^{K_{0} \int_{0}^{t} \mathscr{E}(\eta) d \eta}\left[\tau\left\|A_{0}-\tilde{A}_{0}\right\|^{2}+\left\|\phi_{0}-\tilde{\phi}_{0}\right\|^{2}\right] .
$$

It follows from the last inequality that the weak solution of the problem is unique; moreover, it continuously depends on the initial data.

Hence, we proved the following theorem:

Theorem II.1. The initial boundary value problem (1)-(3) with $c_{1} \in[0,1)$ has a unique weak solution,

$$
A, \phi \in C\left([0, T] ; L^{2}(\Omega)\right) \cap L^{2}\left([0, T] ; H_{0}^{1}(\Omega)\right), \quad \forall T>0,
$$

such that

$$
\|\phi(t)\| \leq M_{0}, \quad\|A(t)\| \leq M_{0}, \quad \forall t>0
$$

and

$$
\int_{0}^{T}\|\nabla \phi(t)\|^{2} d t \leq M_{T}, \quad \int_{0}^{T}\|\nabla A(t)\|^{2} d t \leq M_{T}, \forall T>0
$$

In other words, this problem generates a continuous semigroup $S(t), t \geq 0$, in the phase space $X^{0}:=L^{2}(\Omega) \times L^{2}(\Omega)$. Moreover, (12) implies that this semigroup is bounded dissipative in the phase space $X^{0}$.

Remark II.2 (Ref. 15). In the case $c_{1} \geq 2 c_{2}>0$, multiplying (1) and (2) by $c_{2} A^{*}$ and $2 c_{1} \phi$, respectively, and adding the real parts of the resulting equalities leads to the following estimate:

$$
\begin{aligned}
\frac{d}{d t}\left(\frac{c_{1} \tau}{2}\|A\|^{2}+c_{2}\|\phi\|^{2}\right)-c_{1}\|A\|^{2}+c_{1}\|\nabla A\|^{2} & +c_{1}\|A\|_{L^{4}}^{4} \\
& +2 c_{2} D_{1}\left\|\partial_{x} \phi\right\|^{2}+2 c_{2} D_{1}\left\|\partial_{y} \phi\right\|^{2}+2 c_{2}\left(h, \phi^{2}\right) \leq 0 .
\end{aligned}
$$

Since $\|A\|^{2} \leq\|A\|_{L^{4}}^{4}+\frac{1}{4}|\Omega|$ and $h>0$, we obtain the following analog of (11):

$$
\frac{d}{d t}\left(\frac{c_{1} \tau}{2}\|A\|^{2}+2 c_{2}\|\phi\|^{2}\right)+c_{1}\|\nabla A\|^{2}+2 c_{2} d_{0}\|\nabla \phi\|^{2} \leq \frac{1}{4}|\Omega|
$$

This inequality implies dissipativity of the system in $X^{0}$ when $c_{1} \geq 2 c_{2}>0$.

Thus, the results of Theorem II. 1 are valid also for $c_{1} \geq 2 c_{2}>0$.

\section{GLOBAL ATTRACTOR}

In this section, we prove the existence of a global attractor for the semigroup associated with the initial boundary value problem (1)-(3). In order to establish this result, we rely on the following compactness result:

Lemma III.1. The semigroup $S(t): X^{0} \rightarrow X^{0}, t \geq 0$, is a compact semigroup.

Proof. Multiplying (1) by $-\Delta A^{*}$ in $L^{2}$, then taking $2 R e$ parts of the obtained relation, and utilizing the following inequality:

$$
-2 \operatorname{Re} \int_{\Omega}|A|^{2} A \Delta A^{*} d x d y=4 \int_{\Omega}|\nabla A|^{2}|A|^{2} d x d y+2 \operatorname{Re} \int A^{2}\left(\nabla A^{*}\right)^{2} d x d y \geq 2 \int_{\Omega}|\nabla A|^{2}|A|^{2} d x d y,
$$

we get

$$
\begin{aligned}
\tau \partial_{t}\|\nabla A\|^{2} & -2\|\nabla A\|^{2}+2\|\Delta A\|^{2}+2\left(\phi^{2},|\nabla A|^{2}\right)+2\left(|\nabla A|^{2},|A|^{2}\right) \\
& \leq 4\left|\left(\phi \partial_{x} \phi, A \partial_{x} A^{*}\right)\right|+4\left|\left(\phi \partial_{y} \phi, A \partial_{y} A^{*}\right)\right|+4 c_{1}\left|\left(\phi \partial_{y} A, \Delta A^{*}\right)\right|+2|\beta|\left|\left(A \partial_{y} \phi, \Delta A^{*}\right)\right| .
\end{aligned}
$$

We estimate each term on the right-hand side of (25) by using Cauchy's inequality, Hölder's inequality, and the Ladyzhenskaya inequality given in (18),

$$
\begin{aligned}
4\left|\left(A \partial_{x} A^{*}, \phi \partial_{x} \phi\right)\right| & \leq 4\|\phi\|_{L^{4}}\|\nabla \phi\|_{L^{4}}\|A\|_{L^{4}}\|\nabla A\|_{L^{4}} \\
& \leq 8\|\phi\|^{\frac{1}{2}}\|\nabla \phi\|\|\Delta \phi\|^{\frac{1}{2}}\|A\|^{\frac{1}{2}}\|\nabla A\|\|\Delta A\|^{\frac{1}{2}} \\
& \leq \frac{v_{0}}{8}\|\Delta \phi\|^{2}+\frac{32}{v_{0}}\|\phi\|^{2}\|\nabla \phi\|^{4}+\frac{\|\Delta A\|^{2}}{8}+32\|A\|^{2}\|\nabla A\|^{4} .
\end{aligned}
$$


Repeating exactly the same steps, we estimate the second term on the right-hand side of (25) as follows:

$$
4\left|\left(A \partial_{y} A^{*}, \phi \partial_{y} \phi\right)\right| \leq \frac{v_{0}}{8}\|\Delta \phi\|^{2}+\frac{32}{v_{0}}\|\phi\|^{2}\|\nabla \phi\|^{4}+\frac{\|\Delta A\|^{2}}{8}+32\|A\|^{2}\|\nabla A\|^{4} .
$$

We proceed with estimates of the third and fourth terms,

$$
\begin{aligned}
4 c_{1}\left|\left(\phi \partial_{y} A, \Delta A\right)\right| & \frac{\|\Delta A\|^{2}}{4}+16 c_{1}^{2}\left\|\phi \partial_{y} A\right\|^{2} \\
& \leq \frac{\|\Delta A\|^{2}}{4}+16 c_{1}^{2}\|\phi\|_{L^{4}}^{2}\left\|\partial_{y} A\right\|_{L^{4}}^{2} \\
& \leq \frac{\|\Delta A\|^{2}}{4}+32 c_{1}^{2}\|\phi\|\|\nabla \phi\|\|\nabla A\|\|\Delta A\| \\
& \leq \frac{\|\Delta A\|^{2}}{2}+32^{2} c_{1}^{4}\|\phi\|^{2}\|\nabla \phi\|^{2}\|\nabla A\|^{2}, \\
2\left|\beta \|\left(A \partial_{y} \phi, \Delta A\right)\right| \leq & \frac{\|\Delta A\|^{2}}{4}+4 \beta^{2}\left(|A|^{2},|\nabla \phi|^{2}\right) \\
\leq & \frac{\|\Delta A\|^{2}}{4}+4 \beta^{2}\|A\|_{L^{4}}^{2}\|\nabla \phi\|_{L^{4}}^{2} \\
\leq & \frac{\|\Delta A\|^{2}}{4}+8 \beta^{2}\|A\|\|\nabla A\|\|\nabla \phi\|\|\Delta \phi\| \\
\leq & \frac{\|\Delta A\|^{2}}{4}+\frac{v_{0}}{4}\|\Delta \phi\|^{2}+\frac{64 \beta^{4}}{v_{0}}\|A\|^{2}\|\nabla A\|^{2}\|\nabla \phi\|^{2} .
\end{aligned}
$$

Combining (25) with (26)-(29), we obtain

$$
\begin{aligned}
\tau \frac{d}{d t}\|\nabla A\|^{2}-2\|\nabla A\|^{2}+2\|\Delta A\|^{2}+2\left(\phi^{2},|\nabla A|^{2}\right) & +2\left(|\nabla A|^{2},|A|^{2}\right) \\
\leq & \frac{v_{0}}{2}\|\Delta \phi\|^{2}+\|\Delta A\|^{2}+\frac{64}{v_{0}}\|\phi\|^{2}\|\nabla \phi\|^{4}+64\|A\|^{2}\|\nabla A\|^{4} \\
& +2{ }^{10} c_{1}^{4}\|\phi\|^{2}\|\nabla \phi\|^{2}\|\nabla A\|^{2}+\frac{64 \beta^{4}}{v_{0}}\|A\|^{2}\|\nabla A\|^{2}\|\nabla \phi\|^{2} .
\end{aligned}
$$

On the other hand, multiplying (2) by

$$
-\mathscr{L} \phi=-D_{1} \partial_{x}^{2} \phi-D_{2} \partial_{y}^{2} \phi
$$

and using the following inequality:

$$
v_{0}\|\Delta u\|^{2} \leq\|\mathscr{L} u\|^{2} \leq v_{1}\|\Delta u\|^{2}
$$

which is valid for each $u \in H^{2}(\Omega) \cap H_{0}^{1}(\Omega)$, we obtain

$$
\begin{aligned}
\frac{1}{2} \frac{d}{d t}\left(D_{1}\left\|\partial_{x} \phi\right\|^{2}+D_{2}\left\|\partial_{y} \phi\right\|^{2}\right)+v_{0} & \|\Delta \phi\|^{2}+h D_{1}\left\|\partial_{x} \phi\right\|^{2} \\
& +h D_{2}\left\|\partial_{y}^{2} \phi\right\|^{2} \leq-\left(\phi|A|^{2}, \mathscr{L} \phi\right)+c_{2}\left(\operatorname{Im}\left[A^{*} \partial_{y} A\right], \mathscr{L} \phi\right) .
\end{aligned}
$$

We estimate both terms on the right-hand side of (31) separately,

$$
\begin{aligned}
& \left|\left(\phi|A|^{2}, \mathscr{L} \phi\right)\right| \\
& \leq D_{1}\left|\left(\left(\partial_{x} \phi\right)^{2},|A|^{2}\right)\right|+D_{2}\left|\left(\left(\partial_{y} \phi\right)^{2},|A|^{2}\right)\right|+D_{1}\left|\left(\phi \partial_{x} \phi, \partial_{x}|A|^{2}\right)\right|+D_{2}\left|\left(\phi \partial_{y} \phi, \partial_{y}|A|^{2}\right)\right| \\
& \leq\left(D_{1}+D_{2}\right)\left(|\nabla \phi|^{2},|A|^{2}\right)+2 D_{1}\left(|\phi|\left|\partial_{x} \phi\right|,|A|\left|\partial_{x} A\right|\right) \\
& \quad \leq\left(D_{1}+D_{2}\right)\left(|\nabla \phi|^{2},|A|^{2}\right)+\left(D_{1}+D_{2}\right)\left(\phi^{2},|\nabla \phi|^{2}\right)+\left(D_{1}+D_{2}\right)\left(\left|A^{2}\right|,|\nabla A|^{2}\right) .
\end{aligned}
$$

Estimating each term on the right-hand side of (32) requires the same sequence of arguments, 


$$
\begin{aligned}
&\left(D_{1}+D_{2}\right)\left(|\nabla \phi|^{2},|A|^{2}\right) \leq\left(D_{1}+D_{2}\right)\|\nabla \phi\|_{L^{4}}^{2}\|A\|_{L^{4}}^{2} \\
& \leq 2\left(D_{1}+D_{2}\right)\|\nabla \phi\|\|\Delta \phi\|\|A\|\|\nabla A\| \\
& \leq \varepsilon\|\Delta \phi\|^{2}+\frac{\left(D_{1}+D_{2}\right)^{2}}{\varepsilon}\|\nabla \phi\|^{2}\|A\|^{2}\|\nabla A\|^{2}, \\
&\left(D_{1}+D_{2}\right)\left(\phi^{2},|\nabla \phi|^{2}\right) \leq \varepsilon\|\Delta \phi\|^{2}+\frac{\left(D_{1}+D_{2}\right)^{2}}{\varepsilon}\|\phi\|^{2}\|\nabla \phi\|^{4}, \\
&\left(D_{1}+D_{2}\right)\left(|A|^{2},|\nabla A|^{2}\right) \leq \varepsilon\|\Delta A\|^{2}+\frac{\left(D_{1}+D_{2}\right)^{2}}{\varepsilon}\|A\|^{2}\|\nabla A\|^{4} .
\end{aligned}
$$

Next, we consider the second term on the right-hand side of (31),

$$
\begin{aligned}
\left|c_{2}\left(\operatorname{Im}\left[A^{*} \partial_{y} A\right], \mathscr{L} \phi\right)\right| & \leq \frac{\varepsilon_{1}}{v_{1}}\|\mathscr{L} \phi\|^{2}+\frac{v_{1} c_{2}^{2}}{4 \varepsilon_{1}}\left(|A|^{2},\left|\partial_{y} A\right|^{2}\right) \\
& \leq \varepsilon_{1}\|\Delta \phi\|^{2}+\varepsilon\|\Delta A\|^{2}+\left(\frac{v_{1} c_{2}^{2}}{4 \varepsilon_{1}}\right)^{2} \frac{1}{\varepsilon}\|A\|^{2}\|\nabla A\|^{4} .
\end{aligned}
$$

Equation (31) together with (32)-(36) implies the following estimate:

$$
\begin{aligned}
\frac{1}{2} \frac{d}{d t}\left[D_{1}\left\|\partial_{x} \phi\right\|^{2}+D_{2}\left\|\partial_{y} \phi\right\|^{2}\right]+v_{0}\|\Delta \phi\|^{2}+h D_{1}\left\|\partial_{x} \phi\right\|^{2}+h D_{2}\left\|\partial_{y} \phi\right\|^{2} \\
\leq\left(2 \varepsilon+\varepsilon_{1}\right)\|\Delta \phi\|^{2}+2 \varepsilon\|\Delta A\|^{2}+\left(\frac{v_{1} c_{2}^{2}}{4 \varepsilon_{1}}\right)^{2} \frac{1}{\varepsilon}\|A\|^{2}\|\nabla A\|^{4} \\
+\frac{\left(D_{1}+D_{2}\right)^{2}}{\varepsilon}\left(\|\nabla \phi\|^{2}\|A\|^{2}\|\nabla A\|^{2}+\|\phi\|^{2}\|\nabla \phi\|^{4}+\|A\|^{2}\|\nabla A\|^{4}\right) .
\end{aligned}
$$

We set $\varepsilon=\frac{1}{8}$ and $\varepsilon_{1}=\frac{v_{0}}{4}-\frac{1}{4}$ in (37). Then, adding (30) and (37), we get

$$
\begin{aligned}
\frac{d}{d t}\left[\tau\|\nabla A\|^{2}+D_{1}\left\|\partial_{x} \phi\right\|^{2}+D_{2}\left\|\partial_{y} \phi\right\|^{2}\right] & +v_{0}\|\Delta \phi\|^{2}+\frac{\|\Delta A\|^{2}}{2} \\
& \leq C\left(\|\nabla \phi\|^{2}+\|\nabla A\|^{2}+1\right)\left(\tau\|\nabla A\|^{2}+D_{1}\left\|\partial_{x} \phi\right\|^{2}+D_{2}\left\|\partial_{y} \phi\right\|^{2}\right),
\end{aligned}
$$

where $C$ is a constant that depends on $v_{0}, c_{1}, c_{2}, \beta, D_{1}, D_{2}$, and $M_{0}$, which is a parameter defined in (23).

We multiply (38) by $t$ and rewrite the resulting inequality as follows:

$$
\frac{d}{d t}(t \mathscr{E}(t))-\mathscr{E}(t)+t\left(v_{0}\|\Delta \phi\|^{2}+\frac{\|\Delta A\|^{2}}{2}\right) \leq C\left(\|\nabla \phi\|^{2}+\|\nabla A\|^{2}+1\right) t \mathscr{E}(t)
$$

where

$$
\mathscr{E}(t):=\tau\|\nabla A\|^{2}+D_{1}\left\|\partial_{x} \phi\right\|^{2}+D_{2}\left\|\partial_{y} \phi\right\|^{2} .
$$

Then, we integrate (39) with respect to $t$ over $(0, t)$,

$$
\begin{aligned}
t \mathscr{E}(t)+\int_{0}^{t} \eta\left(v_{0}\|\Delta \phi(\eta)\|^{2}+\frac{\|\Delta A\|^{2}}{2}\right) & d \eta \\
& \leq \int_{0}^{t} \mathscr{E}(\eta) d \eta+C \int_{0}^{t}\left(\|\nabla \phi(\eta)\|^{2}+\|\nabla A(\eta)\|^{2}+1\right) \eta \mathscr{E}(\eta) d \eta
\end{aligned}
$$

The desired estimate follows from the above inequality by first employing the estimate (24) and then the Gronwall lemma,

$$
t \mathscr{E}(t) \leq M_{1}(t) e^{M_{2}(t)},
$$

where $M_{1}(t)$ and $M_{2}(t)$ depend only on $\left\|A_{0}\right\|,\left\|\phi_{0}\right\|$, and $|\Omega|$ as $M_{T}$, which is introduced in (14).

Inequality (40) implies that $\forall t>0$ and $\left[A_{0}, \phi_{0}\right] \in L^{2}(\Omega) \times L^{2}(\Omega)$, 


$$
S(t)\left[A_{0}, \phi_{0}\right] \in H_{0}^{1}(\Omega) \times H_{0}^{1}(\Omega),
$$

i.e., the semigroup $S(t): X^{0} \rightarrow X^{0}, t \geq 0$, generated by the problem (1)-(3) is a compact semigroup.

Thanks to (12), this semigroup is also bounded dissipative. Therefore, the following theorem holds true (see, e.g., Ref. 16).

Theorem III.2. If $c_{1} \in[0,1)$ or $c_{1} \geq 2 c_{2}>0$, then the semigroup

$$
S(t): X^{0} \rightarrow X^{0}, \quad t \geq 0,
$$

generated by the problem (1)-(3) possesses a global attractor $\mathcal{A}$, i.e., a compact, invariant set that attracts uniformly each bounded set of the phase space $X^{0}$.

Moreover, $\mathcal{A}$ is bounded in $H^{1}(\Omega) \times H^{1}(\Omega)$.

Remark III.3. Theorems II.1 and III.2 are also valid under the condition $\left|c_{1}\right|<1$ although the physically relevant case requires $c_{1}$ to be non-negative as stated.

\section{BASIC DYNAMICS}

The purpose of this section is to provide some insight into the behavior of the system of PDEs (1) and (2) by reducing it under special assumptions to systems of ordinary differential equations (ODEs), which can, in turn, be studied as dynamical systems. In particular, we are interested in examining (a) the possibility of pattern formation and (b) the special role that Theorem III.2 gives to the critical value $c_{1}=1$.

\section{A. Polar form}

Starting again from equations (1) and (2), we consider the change of variables $A=\rho e^{i \psi}$ with $\rho=\rho(x, y, t)$ and $\psi=\psi(x, y, t)$. As a preliminary, we note that

$$
\partial_{t} A=e^{i \psi} \partial_{t} \rho+i \rho e^{i \psi} \partial_{t} \psi
$$

(and similarly for the derivatives with respect to $x$ and $y$ ) and

$$
\Delta A=e^{i \psi}\left(\Delta \rho-\rho|\nabla \psi|^{2}\right)+i \rho e^{i \psi}\left(\Delta \psi+\frac{2 \nabla \rho \cdot \nabla \psi}{\rho}\right)
$$

In addition,

$$
\begin{aligned}
-2 i c_{1} \phi \partial_{y} A+i A \partial_{y} \phi=-2 i c_{1} \phi\left(e^{i \psi} \partial_{y} \rho+i \rho e^{i \psi} \partial_{y} \psi\right)+i \beta \rho e^{i \psi} \partial_{y} \phi & \\
& =e^{i \psi}\left(2 c_{1} \rho \phi \partial_{y} \psi\right)+i \rho e^{i \psi}\left(-2 c_{1} \phi \rho^{-1} \partial_{y} \rho+\beta \partial_{y} \phi\right)
\end{aligned}
$$

and

$$
A^{*} \partial_{y} A=\rho e^{-i \psi}\left(e^{i \psi} \partial_{y} \rho+i \rho e^{i \psi} \partial_{y} \psi\right)=\rho \partial_{y} \rho+i \rho^{2} \partial_{y} \psi .
$$

The system then can be written, in terms of the polar variables, as

$$
\begin{aligned}
& \tau \partial_{t} \rho=\Delta \rho-\rho|\nabla \psi|^{2}+\rho+2 c_{1} \rho \phi \partial_{y} \psi-\phi^{2} \rho-\rho^{3}, \\
& \tau \partial_{t} \psi=\Delta \psi+\frac{2 \nabla \rho \cdot \nabla \psi}{\rho}-2 c_{1} \phi \rho^{-1} \partial_{y} \rho+\beta \partial_{y} \phi, \\
& \partial_{t} \phi=\operatorname{div}(D \nabla \phi)-h \phi+\phi \rho^{2}-c_{2} \rho^{2} \partial_{y} \psi,
\end{aligned}
$$

where $D=\left(\begin{array}{cc}D_{1} & 0 \\ 0 & D_{2}\end{array}\right)$.

\section{B. Spatially uniform dynamics}

We study first the case where all the variables are constant in space, i.e., $\rho=\rho(t), \psi=\psi(t)$, and $\phi=\phi(t)$. Equivalently, this can be thought of as a result of performing the rescaling $x \rightarrow x / \varepsilon, y \rightarrow y / \varepsilon$ and dropping the higher order terms with respect to $\varepsilon$. In any case, we end up with the following reduced system: 


$$
\begin{aligned}
& \tau \partial_{t} \rho=\rho\left(1-\phi^{2}-\rho^{2}\right), \\
& \tau \partial_{t} \psi=0, \\
& \partial_{t} \phi=\phi\left(\rho^{2}-h\right) .
\end{aligned}
$$

It follows immediately that the phase $\psi$ of $A$ is decoupled from the rest of the system and can be ignored. The dynamics of the reduced system (46) and (48) depend on the value of the dampening parameter $h$ :

1. Figure 4 (left), $h=0$ : There are two saddle points at $(\rho, \phi)=( \pm 1,0)$. The line segment $\rho=0, \phi \in(-1,1)$ consists of degenerate unstable critical points, whereas the rest of the $\phi$ axis is made of stable degenerate critical points.

2. Figure 4 (right), $0<h<1$ : There are three saddle points at $(\rho, \phi)=( \pm 1,0)$ and $(0,0)$ and four spiral sinks on the unit circle at $( \pm \sqrt{h}, \pm \sqrt{1-h})$. Each quadrant converges to the corresponding sink.

3. Figure 5 (left), $h=1$ : There is a saddle point at $(\rho, \phi)=(0,0)$ and two degenerate stable critical points at $( \pm 1,0)$. Points in each half-space $\phi<0$ and $\phi>0$ converge to the corresponding critical point.

4. Figure 5 (right), $h>1$ : Similar behavior to the critical case $h=1$.

Based on these cases, we expect the possibility of pattern formation in the case when $0<h<1$, where there are two (given that $\rho$ is, in fact, the modulus $|A|$ of the complex number $A$, we are effectively only interested in the positive half-plane $\rho \geq 0$ ) distinct non-trivial critical points $(\rho, \phi)=(\sqrt{h}, \pm \sqrt{1-h})$.

\section{Reduced dynamics in the presence of a phase gradient}

To reintroduce the phase variable $\psi$ into the dynamics, we assume as before that $\rho=\rho(x / \varepsilon, y / \varepsilon, t)$ and $\phi=\phi(x / \varepsilon, y / \varepsilon, t)$, but $\psi=\psi(x / \varepsilon, y, t / \varepsilon)$, i.e., the variation of $\psi$ in the $y$ direction is significant. This leads to the reduced system

$$
\begin{aligned}
& \tau \partial_{t} \rho=\rho\left(-\left|\partial_{y} \psi\right|^{2}+1+2 c_{1} \phi \partial_{y} \psi-\phi^{2}-\rho^{2}\right) \\
& \partial_{y}^{2} \psi=0 \\
& \partial_{t} \phi=\phi\left(\rho^{2}-h\right)-c_{2} \rho^{2} \partial_{y} \psi
\end{aligned}
$$
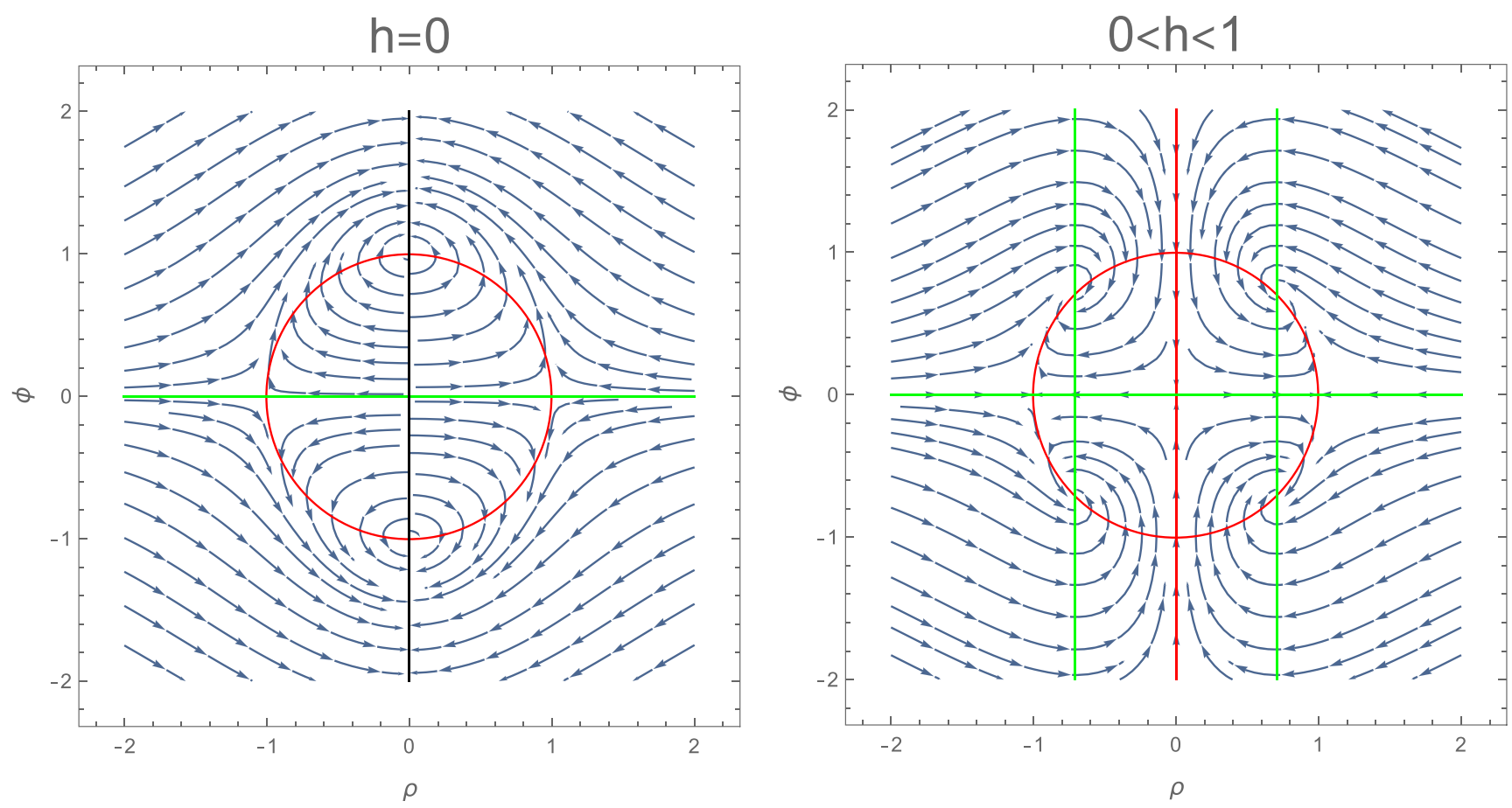

FIG. 4. Phase diagrams for the spatially uniform case with dampening parameter $h=0$ (left) and $0<h<1$ (right). 

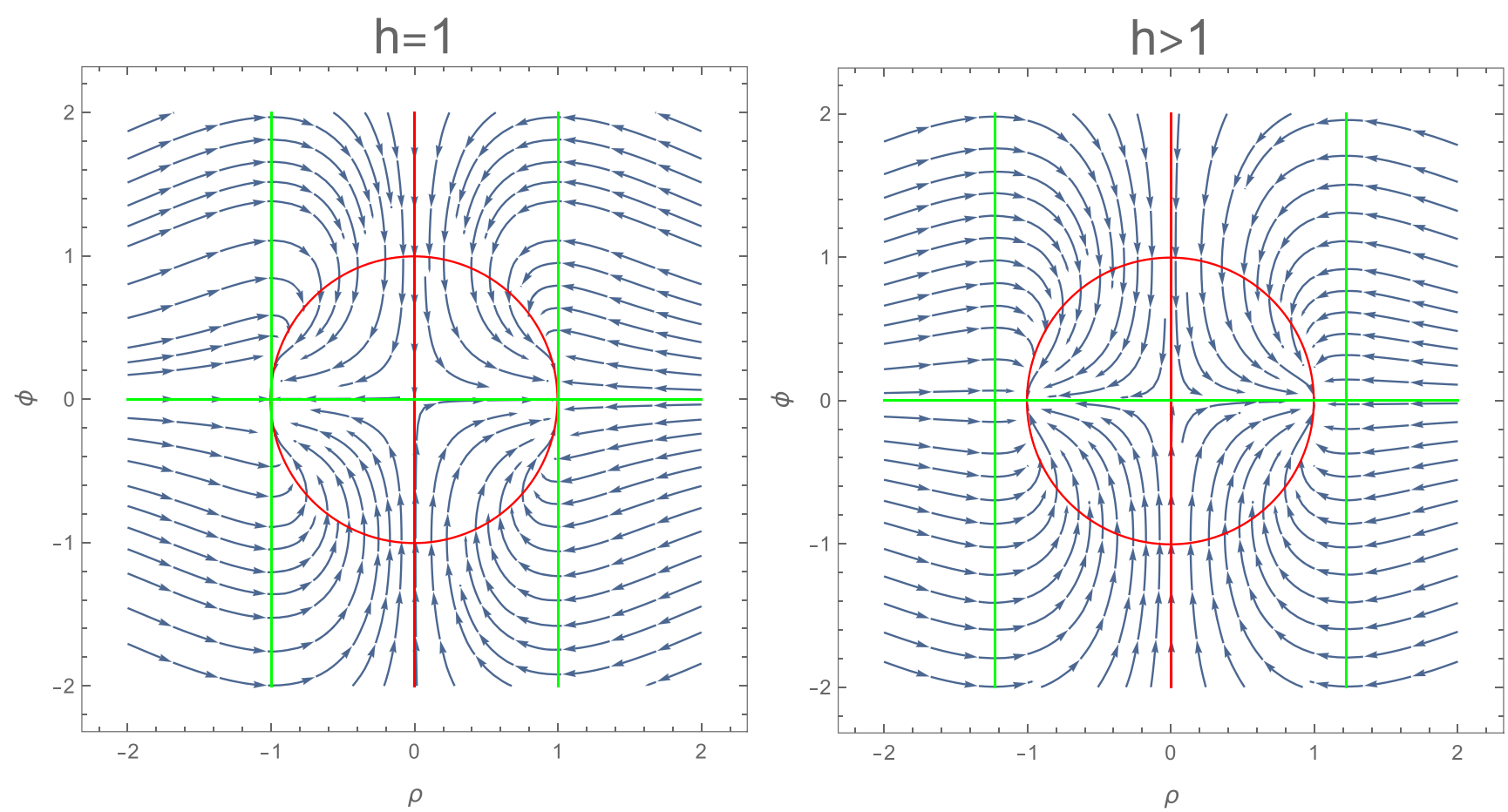

FIG. 5. Phase diagrams for the spatially uniform case with critical dampening parameter $h=1$ (left) and $h>1$ (right).

Because of the second equation, the derivative $\partial_{y} \psi$ is constant. Making the change of variables $\chi=\partial_{y} \psi=$ const., we end up with the following system of ODEs:

$$
\begin{aligned}
& \tau \frac{d \rho}{d t}=\rho\left[\left(1-\rho^{2}\right)-\left(\phi-c_{1} \chi\right)^{2}-\left(1-c_{1}^{2}\right) \chi^{2}\right], \\
& \frac{d \phi}{d t}=-h \phi+\rho^{2}\left(\phi-c_{2} \chi\right) .
\end{aligned}
$$

The locus of the critical points of this dynamical system, for a given phase gradient $\chi$, is the set

$$
C_{\chi}=\left\{(\rho, \phi) \mid \phi=\frac{c_{2} \chi \rho^{2}}{\rho^{2}-h}\right\} \cap\left\{(\rho, \phi) \mid \rho^{2}+\left(\phi-c_{1} \chi\right)^{2}=1+\left(c_{1}^{2}-1\right) \chi^{2} \vee \rho=0\right\}
$$

The rational curve $\phi=\frac{c_{2} \chi \rho^{2}}{\rho^{2}-h}$ has three branches, with asymptotes at $\rho= \pm \sqrt{h}$ and $\phi=c_{2} \chi$, whereas the second set is the union of a circle with center $\left(0, c_{1} \chi\right)$ and radius $\sqrt{1+\left(c_{1}^{2}-1\right) \chi^{2}}$ and the line $\rho=0$. The set always includes the point $(\rho, \phi)=(0,0)$ and, depending on the values of the parameters $c_{1}$ and $c_{2}$ and the phase gradient $\chi$, up to 3 more critical points in the positive half-plane $\rho \geq 0$, where the branches of the rational curve intersect the circle.

The qualitative difference in the dynamics, between the subcritical $0 \leq c_{1}<1$ and supercritical $c_{1}>1$ cases, appears to be due to the location and size of the aforementioned circle on the phase diagram (see Figs. 6 and 7 ) and the induced presence or absence of non-trivial critical points. More specifically, for small values of the phase gradient, $\left|\partial_{t} \psi\right| \ll 1$, the circle is centered near the origin and has radius $\sim 1$, yielding dynamics similar to the ones presented in Sec. IV B. As the phase gradient $\chi$ increases in magnitude in the case $0 \leq c_{1}<1$, the radius $\sqrt{1+\left(c_{1}^{2}-1\right) \chi^{2}}$ is decreasing until eventually $1+\left(c_{1}^{2}-1\right) \chi^{2}<0$, and there are no other critical points, but the origin (Fig. 6) to which all orbits are attracted. On the other hand, when $c_{1}>1$, the radius of the circle increases with higher values of $\left|\partial_{t} \psi\right|$ and there is always at least one more critical point apart from the origin (Fig. 7).

Remark IV.1. In Chap. 2 of Ref. 8, one can find a more in-depth stability analysis for solutions of the form $A=\rho e^{i(Q x+P y)}$ for the isotropic case $c_{1}=c_{2}=1$. 

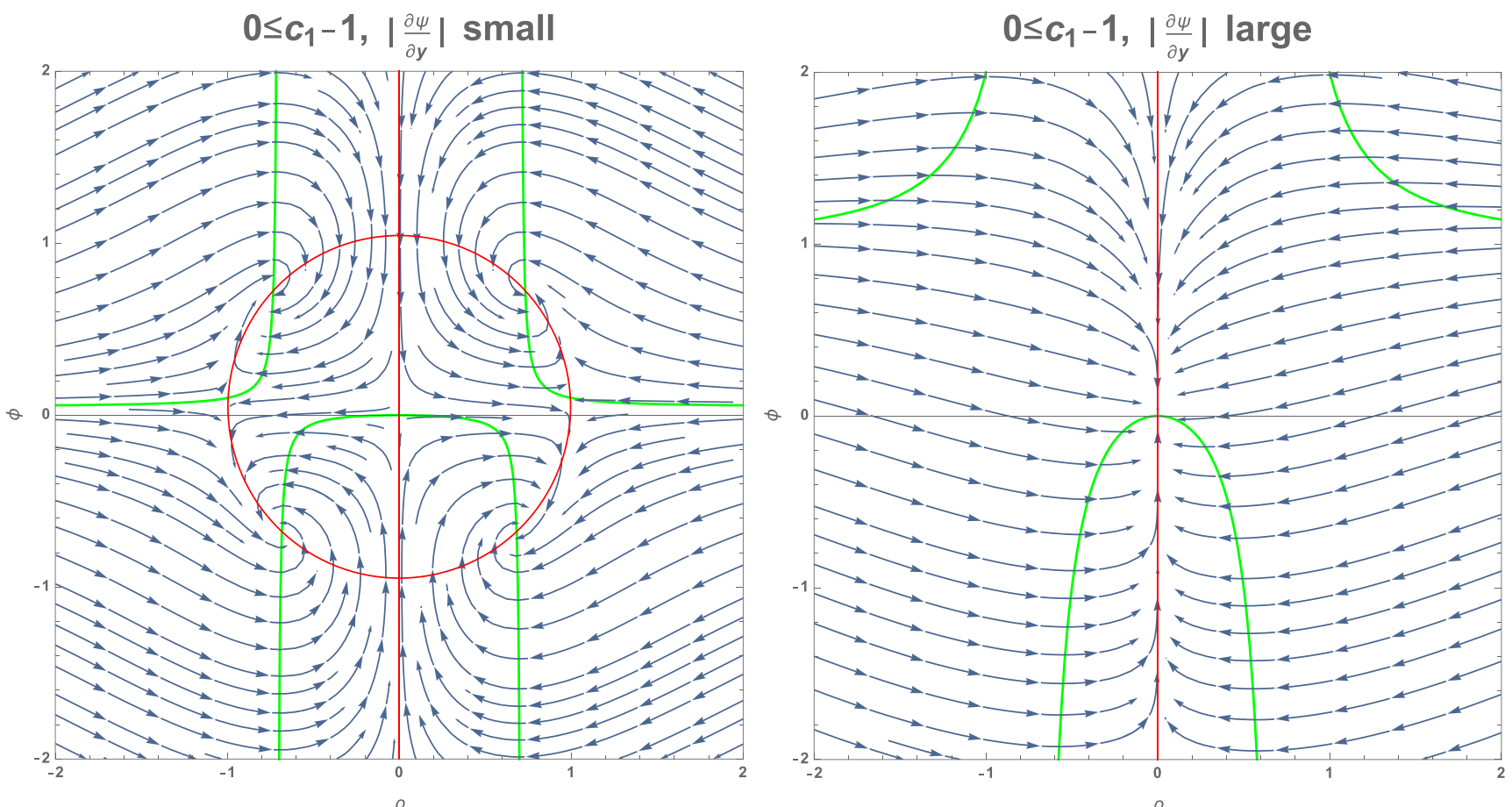

FIG. 6. Phase diagram in the case of $0 \leq c_{1}<1$ (and $0<h<1$ ) and for different values of the phase gradient $\partial_{y} \psi$.
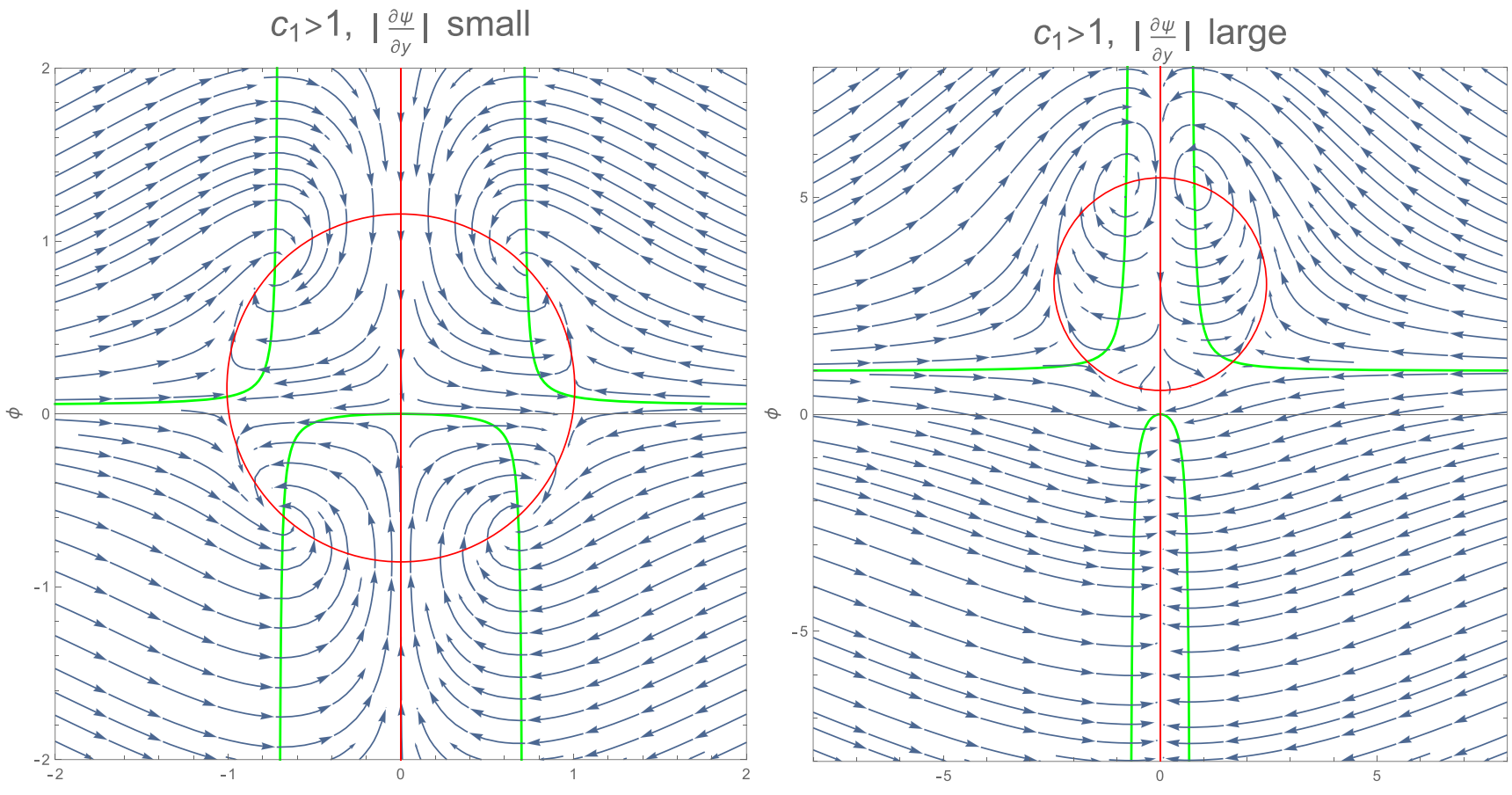

FIG. 7. Phase diagram in the case of $c_{1}>1$ (and $0<h<1$ ) and for different values of the phase gradient $\partial_{y} \psi$. 
Remark IV.2. The results obtained in this work hold true for the case of Neumann boundary conditions as well as periodic boundary conditions, since the parameter $h$ in equation (2) is positive and equation (1) involves the term $|A|^{2} A$. In this case, instead of the inequality (18), it is necessary to use the following inequality:

$$
\|u\|_{L^{4}}^{2} \leq C\|u\|\|u\|_{H^{1}}
$$

which is valid for each $u \in H^{1}(\Omega)$.

\section{DATA AVAILABILITY}

Data sharing is not applicable to this article as no new data were created or analyzed in this study.

\section{REFERENCES}

${ }^{1}$ G. H. Heilmeier and W. Helfrich, “Orientational oscillations in nematic liquid crystals,” Appl. Phys. Lett. 16, 155-157 (1970).

${ }^{2}$ O. L. C. Group, “AC and DC regimes of the electrohydrodynamic instabilities in nematic liquid crystals,” Mol. Cryst. Liq. Cryst. 12, 251-266 (1971).

${ }^{3}$ T. Börzsönyi, Á. Buka, A. P. Krekhov, and L. Kramer, "Response of a homeotropic nematic liquid crystal to rectilinear oscillatory shear,” Phys. Rev. E 58, 7419 (1998).

${ }^{4}$ A. Buka and L. Kramer, Pattern Formation in Liquid Crystals (Springer Science \& Business Media, 2012).

${ }^{5}$ G. Demeter, "Complex nonlinear behavior in optically excited nematic liquid crystals," Phys. Rev. E 61, 6678 (2000).

${ }^{6}$ L. Kramer and W. Pesch, "Electrohydrodynamic instabilities in nematic liquid crystals," in Pattern Formation in Liquid Crystals (Springer, New York, 1996 ), pp. 221-255.

${ }^{7}$ M. A. Scherer, G. Ahlers, F. Hörner, and I. Rehberg, "Deviations from linear theory for fluctuations below the supercritical primary bifurcation to electroconvection," Phys. Rev. Lett. 85, 3754 (2000).

${ }^{8}$ A. G. Rossberg, “The amplitude formalism for pattern forming systems with spontaneously broken isotropy and some applications," Ph.D. thesis, Universität Bayreuth, Fakultät für Mathematik, Physik und Informatik, 1998.

${ }^{9}$ A. G. Rossberg, A. Hertrich, L. Kramer, and W. Pesch, "Weakly nonlinear theory of pattern-forming systems with spontaneously broken isotropy," Phys. Rev. Lett. 76, 4729 (1996).

${ }^{10}$ J.-H. Huh, Y. Hidaka, A. G. Rossberg, and S. Kai, "Pattern formation of chevrons in the conduction regime in homeotropically aligned liquid crystals," Phys. Rev. E 61, 2769 (2000).

${ }^{11}$ S. Tatsumi, M. Sano, and A. G. Rossberg, “Observation of stable phase jump lines in convection of a twisted nematic liquid crystal,” Phys. Rev. E 73, 011704 (2006).

${ }^{12}$ S. Komineas, H. Zhao, and L. Kramer, "Modulated structures in electroconvection in nematic liquid crystals,” Phys. Rev. E 67, 031701 (2003).

${ }^{13}$ H. Sakaguchi and A. Matsuda, "Chevron patterns and defect lattices in an anisotropic model for electroconvection,” Physica D 238, 1-8 (2009).

${ }^{14}$ A. G. Rossberg and L. Kramer, "Pattern formation from defect chaos-A theory of chevrons," Physica D 115, 19-28 (1998).

${ }^{15}$ E. S. Titi, oral communication (2020).

${ }^{16}$ R. Temam, Infinite-Dimensional Dynamical Systems in Mechanics and Physics (Springer Science \& Business Media, 2013 ), Vol. 68. 